\title{
Ergebnisse einer sechsmonatigen Doppelblindstudie Vergleichsstudie zwischen Amisulprid und Risperidon
}

\begin{abstract}
Quelle: Sechter D, Peuskens J, Fleurot $O$, Rein W, Lecrubier Y; Amisulpride Study Group. Amisulpride vs. risperidone in chronic schizophrenia: results of a 6-month double-blind study. Neuropsychopharmacology 2002; 27(6): 1071-1081
\end{abstract}

\section{Zusammenfassung}

Die sechsmonatige randomisierte Doppelblindstudie verglich Wirksamkeit und Verträglichkeit der beiden atypischen Antipsychotika Amisulprid und Risperidon. 309 Patienten erhielten sechs Monate entweder Amisulprid (400-1000 mg/Tag) oder Risperidon (4-10 mg/Tag). In beiden Gruppen verbesserte sich die Symptomatik vergleichbar, wie anhand Positive and Negative Syndrome Scale (PANSS), Brief Psychiatric Rating Scale (BPRS), PANSS-Positiv-Subskala und Bech Rafaelsen Melancholia Skala (BRMS) im Vergleich zu den Ausgangswerten beurteilt wurde. Unter Amisulprid sprachen signifikant mehr Patienten auf die Behandlung an als unter Risperidon. Therapieresponse war dabei definiert als $\geq 50 \%$ Verbesserung auf der PANSS- und BPRSGesamtskala oder „sehr stark/stark verbessert“ auf der Clinical Global Impression (CGI)-Skala.

Außerdem zeigten die Patienten, die Amisulprid erhalten hatten, eine signifikant stärkere Verbesserung bezüglich des sozialen bzw. beruflichen Funktionsniveaus (Social and Occupational Functioning Assessment Scale) und wurden auch subjektiv besser eingestuft als die Patienten der Risperidongruppe.

In beiden Behandlungsgruppen wurde die Behandlung gut toleriert, die Inzidenz extrapyramidaler Störungen war vergleichbar niedrig. In der Amisulpridgruppe traten seltener Gewichtszunahmen und endokrine/sexuelle Störungen aus.

\section{Studiendesign}

Multizentische, doppelblind randomisierte Vergleichsstudie über einen Zeitraum von sechs Monaten

\section{Patienten}

310 erwachsene Patienten (Alter zwischen 18 und 65 Jahren), die die DSM-IV-Kriterien für die Diagnose Schizophrenie erfüllten. Die behandlungsbedürftigen Patienten waren mindestens seit zwei Jahren erkrankt und wiesen bei Studienbeginn einen PANSS-Score zwischen 60 und 120 auf.

\section{Behandlung}

Nach einer sechstägigen Washout-Phase mit Plazebo erhielten die Patienten initial $400 \mathrm{mg} /$ Tag Amisulprid ( $\mathrm{n}=152)$, ab Tag 2 bis Tag 7600
mg/Tag Amisulprid bzw. am 1. Behandlungstag $1 \mathrm{mg} /$ Tag Risperidon ( $n=158$ ), die Risperidondosis wurde bis zum 4. Behandlungstag auf $4 \mathrm{mg}$ aufdosiert. Ab der 2. Behandlungswoche erhielten die Patienten 400-1000 mg/Tag Amisulprid bzw. 4-10 mg/Tag Risperidon.

Als Zusatzmedikation waren bei Bedarf Biperiden, Benzodiazepine (in der 1. Behandlungswoche bis zu 40 mg/Tag Diazepam-Äquivalenten, ab der 2. Behandlungswoche bis zu 30 mg/Tag Diazepam-Äquivalenten, insgesamt aber nicht länger als vier Wochen) sowie Nicht-Benzodiazepinderivate (Zolpidem $\leq 10$ mg/Tag oder Zopiclon $\leq 7,5$ mg/Tag) erlaubt.

Fazit

Amisulprid und Risperidon sind über einen Zeitraum von sechs Monaten vergleichbar wirksam und sicher. Bezüglich PANSS und BPRS zeigte sich kein statistischer Unterschied zwischen beiden Behandlungsgruppen.

In der Amsulpridgruppe sprachen signifikant mehr Patienten auf die Behandlung an als unter Risperidon. Auch hinsichtlich Gewichtszunahme sowie endokriner/sexueller Störungen schnitten die Patienten unter Amisulprid deutlich besser ab als unter Risperidon.

\section{Tab. 1 Ergebnis der Vergleichsstudie}

D PANSS-Gesamtwert
D BPRS-Gesamtwert
D CGI
D PANSS-Positivscore
D PANSS-Negativscore
D BRMS
Responserate (Verbesserung $\geq 50 \%$ PANSS-Gesamtwert)
Responserate (Verbesserung $\geq 50 \%$ BPRS-Gesamtwert)
Responserate („sehr stark/stark verbessert“ auf der CGI-Skala)
Responserate (Verbesserung $\geq 30 \%$ SOFAS)
Subjektive Einstufung der Verbesserung
Gewichtsveränderung $\geq 7 \%$
EPS

Endokrine Störungen

\section{Amisulprid ( $\mathrm{n}=121)$}

32,2

19,8

1,7

11,8

5,1

4,9

$65,3 \%$

$71,9 \%$

$76,9 \%$

$49 \%$

$93 \%$

$18 \%$

11,85

$0,7 \%$

\begin{tabular}{c} 
Risperidon (n=123) \\
\hline 31,4 \\
19,6 \\
1,5 \\
12,1 \\
3,9 \\
3,9 \\
$52 \%$ \\
$57,7 \%$ \\
$65 \%$ \\
$35 \%$ \\
$83 \%$ \\
$34 \%$ \\
$17,1 \%$ \\
$5,7 \%$ \\
\hline
\end{tabular}

Signifikanz

ns ns ns

ns

ns

ns

$p=0,036$

$p=0,02$

$p=0,042$

$p=0,033$

$\mathrm{p}=0,015$

$p=0,026$

ns

$p<0,01$ 